\title{
Alternate-current dynamic reorganization and nonequilibrium phase transition in driven vortex matter
}

\author{
M. Marziali Bermúdez, ${ }^{1,2}$ L. F. Cugliandolo, ${ }^{3,4}$ and G. Pasquini ${ }^{1,5, *}$ \\ ${ }^{1}$ Departamento de Física, Facultad de Ciencias Exactas y Naturales, Universidad de Buenos Aires, C1428EGA Buenos Aires, Argentina \\ ${ }^{2}$ Instituto de Física de Líquidos y Sistemas Biológicos (IFLYSIB), UNLP-CONICET, B1900BTE La Plata, Argentina \\ ${ }^{3}$ Laboratoire de Physique Théorique et Hautes Energies, Sorbonne Université, CNRS UMR 7589, 4 Place Jussieu, \\ Tour 13, 5ème étage, 75252 Paris Cedex 05, France \\ ${ }^{4}$ Institut Universitaire de France, 1 rue Descartes, 75231 Paris Cedex 05, France \\ ${ }^{5}$ Instituto de Física de Buenos Aires (IFIBA), UBA-CONICET, C1428EGA Buenos Aires, Argentina
}

(Received 18 September 2019; revised manuscript received 12 December 2019; accepted 28 January 2020; published 10 February 2020)

\begin{abstract}
Externally driven glassy systems may undergo nonequilibrium phase transitions (NEPTs). In particular, acdriven systems display special features, such as those observed in the vortex matter of $\mathrm{NbSe}_{2}$, where oscillatory drives reorganize the system into partially ordered vortex lattices. We provide experimental evidence for this dynamic reorganization and we show an unambiguous signature of its connection with an NEPT driven by ac forces. We perform a scaling analysis and we estimate critical exponents for this transition. Our results share similarities with some glass-to-viscous-liquid NEPTs and invite a search for similar physics in other elastic disordered media.
\end{abstract}

DOI: 10.1103/PhysRevB.101.060502

Glassiness is a synonym of rich dynamics, closely related to metastability and history effects in which plasticity may play a key role. Indeed, when externally driven, glassy systems may adopt self-organized configurations and undergo nonequilibrium phase transitions (NEPTs) [1-3]. Vortex matter in type-II superconductors shows aspects of glassiness. These systems are often modeled as disordered elastic media, a category which also includes magnetic $[4,5]$ and ferroelectric $[6,7]$ domain walls, as well as interacting particles in disordered substrates such as colloidal systems $[3,8,9]$, Wigner crystals $[3,10]$, or skyrmion lattices $[3,11]$. Although the microscopic equations behind these systems are completely different, under some reliable assumptions all of them can be described as elastic manifolds in a disordered landscape [12-14]. In all these cases, depinning occurs when an external continuous drive is increased beyond a critical value. The nature of this transition and its relation to the proliferation or annealing of topological defects have been thoroughly studied, though many questions still remain unsolved in this field [3].

The dynamics of glassy systems driven by alternating (ac) forces have received much less attention. Work carried out mostly during the last decades showed that ac-driven systems display special features, not directly translatable from the corresponding dc regimes, and may be dynamically reorganized into different configurations [15-22]. In particular, molecular dynamics simulations of ac-driven two-dimensional (2D) vortex lattices (VLs) revealed plastic ac depinning in the lowfrequency regime, when the driving ac Lorentz force $F_{\mathrm{L}}$ over-

*pasquini@df.uba.ar comes a critical force $F_{\mathrm{c}}$ and moves vortices over distances larger than the typical pinning radius in each ac cycle [19]. For $F_{\mathrm{L}}<F_{\mathrm{c}}$, plastic random displacement produces a huge number of VL dislocations, but most vortices remain trapped around the pinning sites and the final configuration depends strongly on the initial conditions. On the contrary, above $F_{\mathrm{c}}$, the memory of the initial configuration is lost after a transient number of cycles $\left(N_{\mathrm{ac}}\right)$ that depends on the amplitude and frequency of $F_{\mathrm{L}}$. Past the transient, the density of VL dislocations and the mean vortex velocity remain fluctuating around stationary values which may still depend on the parameters of the ac drive [19]. These dynamic steady states are reminiscent of the "fluctuating steady states" observed in colloidal systems $[8,9]$.

Driven 2D VLs can be used as simple models for a small portion of the 3D vortex systems studied experimentally, for which history effects in the response have been often observed [20] and signatures of criticality at the depinning transition were reported [3,23]. A prototype of such systems is the vortex matter in clean $\mathrm{NbSe}_{2}$ single crystals in which the stable vortex phase at low temperatures and weak magnetic fields is an ordered Bragg glass (BG) without VL dislocations [24]. When field cooled (FC) from the normal state, the system is trapped in disordered metastable configurations where the VL is strongly pinned [21,25]. However, by applying high transport current densities [25-27] or large oscillatory shaking magnetic fields $[18,20,21]$, the system overcomes energy barriers and reaches the ordered BG with lower effective pinning. With increasing field and/or temperature, the system undergoes an order-disorder transition to a disordered glass, whose fingerprint is the anomaly known as the peak effect (PE) [28-30], consisting in a sudden increase of the 


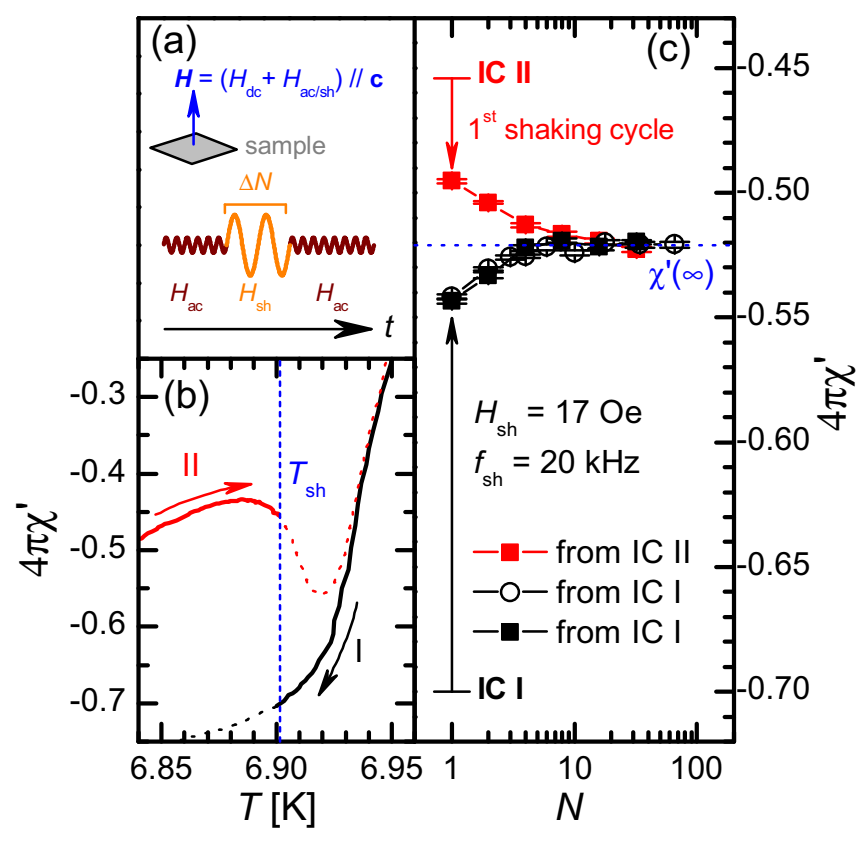

FIG. 1. (a) Experimental setup: The dc field $\left(\mathbf{H}_{\mathrm{dc}}\right)$ is parallel to $\mathbf{H}_{\mathrm{sh}}$ and $\mathbf{H}_{\mathrm{ac}}$, aligned with the $c$ axis. At selected times, the $\chi$ measurement is interrupted to apply a burst of $\Delta N$ shaking pulses. (b) In-phase component of $\chi^{\prime}(T)$ in the PE region, corresponding to field cooling a disordered VL (black) and warming an ordered VL (red). Solid lines show the evolution up to $T_{\mathrm{sh}}$ (vertical dotted line), the stable temperature during shaking; without shaking, $\chi^{\prime}(T)$ would follow the dotted lines. (c) Evolution of $\chi^{\prime}$, after applying $N$ shaking pulses starting from the IC I (black) and IC II (red). After a transient number of cycles $N_{\mathrm{ac}}$, an IC-independent value $\chi_{\infty}^{\prime}$ is reached.

effective pinning. Intermediate responses that broaden the PE have been ascribed in part to surface contamination induced by the probing transport current [27]. However, combined ac susceptibility and small-angle neutron scattering experiments support the existence of a narrow region between the ordered and the disordered phases $[18,31,32]$ where the application of shaking magnetic fields gives rise to bulk VL configurations with intermediate degrees of disorder, correlated with intermediate vortex responses [21]. A consistent scenario also emerges from tunneling spectroscopy experiments carried out in the PE region of Co-doped $\mathrm{NbSe}_{2}$ single crystals [33].

In this Rapid Communication, we present experimental results that show that these "intermediate" configurations are originated from a VL reorganization driven by the oscillatory dynamics. Moreover, unambiguous signatures of criticality suggest that this reorganization is closely associated with a NEPT, possibly related with the ac vortex depinning.

The vortex response may be accessed in different ways. The best choice, given the purpose of the present work, is to record the linear ac susceptibility $\chi^{\prime}$ with a noninvasive measurement [20,21]. In our experiments, this is achieved with the setup and procedure sketched in Fig. 1(a): A permanent dc field $\mathbf{H}_{\mathrm{dc}}$, applied on a superconducting single crystal, generates a vortex arrangement, which is prepared in an initial (history-dependent) configuration. At selected times, the ac driving field, that we call the shaking field $\mathbf{H}_{\text {sh }}$, is switched on, as a way to reorganize the vortex configuration. Subsequently, the shaking field is switched off. Before and after shaking the system, the linear response is measured by applying a very small ac field $\mathbf{H}_{\mathrm{ac}}$ that forces vortices to perform small (harmonic) oscillations inside their effective pinning potential wells, without modifying their spatial configuration. These oscillations propagate through the sample due to the vortexvortex repulsion, with a characteristic penetration depth $\lambda_{\text {ac }}$ that, for a fixed experimental geometry, determines the linear ac susceptibility [34]. In the low-frequency Campbell regime [35], vortices oscillate, in a mean-field approximation [36], in phase with $\mathbf{H}_{\mathrm{ac}}$. In this case, $\lambda_{\mathrm{ac}}$ is related to the curvature of the effective pinning potential and determines the in-phase inductive component of $\chi^{\prime}$, that is nearly frequency independent.

We used a clean $1 \times 1 \times 0.2 \mathrm{~mm}^{3} \mathrm{NbSe}_{2}$ single crystal with $T_{\mathrm{c}}=7.2 \mathrm{~K}$ grown at Bell Laboratories [37]. The phase diagram for several crystals from this source shows only minor variations, also compared to other samples reported in the literature. Linear ac susceptibility measurements were done using a homemade susceptometer based on the mutual inductance technique, installed in a cryostat that allows temperature regulation within $\Delta T \leqslant 2 \mathrm{mK}$. In our setup, the dc field $\left(H_{\mathrm{dc}}=1 \mathrm{kOe}\right)$ is parallel to the perturbation field $\left(H_{\mathrm{ac}}=10 \mathrm{mOe}\right)$ and the $c$ axis of the sample [Fig. 1(a)]. With these parameters, the linear response, low dissipation, and frequency independence-characteristic features of the linear Campbell regime-were verified. We then chose to use $f_{\text {ac }}=90 \mathrm{kHz}$ to have a good resolution.

Shaking fields were applied parallel to the dc field by controlling the number, amplitude, and period of current pulse trains applied to the primary circuit. Local self-heating induced by the dissipation of ac shaking current inside the sample at high shaking amplitudes and/or frequencies was avoided by applying temporally spaced short bursts. Because the order-disorder transition region is characterized by a sharp increase in the ratio between effective pinning and elastic forces with temperature, the system's behavior is strongly temperature dependent. Therefore, good control over the temperature was assured.

Figure 1(b) displays different linear ac susceptibility responses in the PE region. The black curve shows the response measured during a FC procedure, where the VL is disordered and strongly pinned. The red curve displays the response of an ordered, weakly pinned VL, recorded in a warming procedure, after having shaken the system with 1000 cycles of a large ac field $(24 \mathrm{Oe}, 1 \mathrm{kHz})$ at low temperature $(\sim 6.5 \mathrm{~K})$. These responses, well defined and repeatable under the same cooling/warming protocols, can be used as different initial conditions (ICs) for our experiments. Condition I corresponds to the disordered FC VL and condition II to the ordered VL. Once an IC is selected, a temperature $T_{\mathrm{sh}}$ in the PE region is chosen and made stable.

In the absence of any additional driving force, there is no significant evolution of the susceptibility with time and the system remains mainly trapped near these ICs. However, as observed in other complex systems [38,39], large oscillatory driving forces may dynamically assist the system to evolve towards a stationary state, independent of the IC, but strongly temperature dependent [18]. Hence, we chose $T_{\mathrm{sh}}=6.9 \mathrm{~K}$, where the stationary state is conveniently distant to both ICs. 


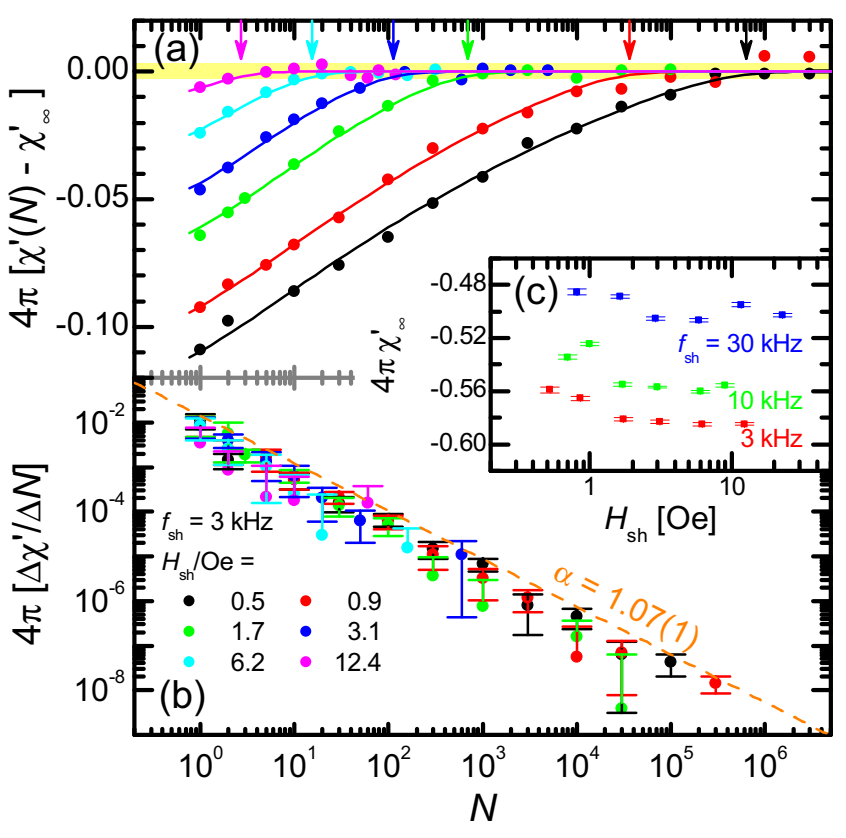

FIG. 2. (a) Difference between the in-phase linear response after $N$ shaking pulses $\chi^{\prime}(N)$ and its asymptotic value $\chi_{\infty}^{\prime}$ for different shaking amplitudes $H_{\text {sh }}$ at $f_{\text {sh }}=3 \mathrm{kHz}$ (log-linear scale). Continuous lines are the functions in Eq. (3) and the vertical arrows indicate the characteristic number of cycles $N_{\mathrm{ac}}$. (b) Finite difference approximation to the derivative of the functions above, against $N$, in double-log scale. The slope of the continuous orange line yields the critical exponent $\alpha$. (c) $\chi_{\infty}^{\prime}$ vs $H_{\mathrm{sh}}$ for several $f_{\mathrm{sh}}$.

We move then to the proper dynamic measurements of our interest. After stabilizing the temperature, we apply a series of pulses of amplitude $H_{\mathrm{sh}}$ and period $1 / f_{\mathrm{sh}}$, and we measure $\chi$ after $1,2, \ldots, N$ pulses. The resulting in-phase component as a function of the number of pulses $N$ is shown in Fig. 1(c): with red squares data for an IC in branch II, whereas with black symbols two examples of the evolution starting from condition I [see Fig. 1(b)]. The effect of the first pulse depends strongly on the IC. However, after a transient number of cycles that we call $N_{\mathrm{ac}}$, the responses converge to a common stationary value $\chi_{\infty}^{\prime}$. We have checked that the evolution $\chi^{\prime}(N)$ does not depend on the number of pulses conforming each applied shaking burst and that it is not modified by the very weak perturbation used during the measurements.

Both the evolution and stationary response may depend on the amplitude $H_{\text {sh }}$ and frequency $f_{\text {sh }}$ of the shaking field. The variation of $\chi_{\infty}^{\prime}$ as a function of $H_{\mathrm{sh}}$ is displayed for selected $f_{\text {sh }}$ in the inset of Fig. 2(c). On the one hand, the asymptotic value increases with $f_{\mathrm{sh}}$ at frequencies higher than $3 \mathrm{kHz}$ but is independent of the shaking frequencies at low $f_{\text {sh }} \lesssim 1 \mathrm{kHz}$ [20]. On the other hand, at a fixed $f_{\text {sh }}$, the final response is nearly $H_{\text {sh }}$ independent, as well as independent of the IC, for $H_{\mathrm{sh}}>1 \mathrm{Oe}$, although it keeps its frequency dependence even for these large field amplitudes. Therefore, quite generally, the driven vortex system evolves to different dynamically organized stationary configurations. Whether the response easily converges to a final value independent of the ICs depends on the shaking amplitude $H_{\mathrm{sh}}$, which determines the typical vortex displacements.
Figure 2(a) shows an example of the evolution of $\chi^{\prime}$ with the number of applied pulses $N$ tending to the stationary response $\chi_{\infty}^{\prime}$, for different amplitudes ranging from 0.5 to $12 \mathrm{Oe}$, at $f_{\mathrm{sh}}=3 \mathrm{kHz}$. A quick and good convergence to $\chi_{\infty}^{\prime}$ in a characteristic number of cycles $N_{\text {ac }} \lesssim 1000$ is achieved for $H_{\mathrm{sh}} \gtrsim 1 \mathrm{Oe}$, that completely penetrates the sample, for which we can estimate a vortex displacement larger than the VL parameter in most of the sample [see the Supplemental Material (SM) in Ref. [21]]. However, with decreasing amplitudes, $N_{\text {ac }}$ increases by several orders of magnitude, beyond the experimentally accessible range, suggesting a divergence. A possible origin for this divergence could be the fact that the shaking field plays the role of an "effective temperature" in an activated process, where activation barriers grow when approaching the stable state [40]. We have tested that our data are not compatible with the expected behavior [41] and we therefore discarded this possibility. On the other hand, a divergent $N_{\mathrm{ac}}$ at a critical shaking amplitude $H_{\mathrm{sh}}^{c}$ could be indicative of a dynamic phase transition. We explore the latter possibility in the following.

In case we were confronted to a NEPT, a critical behavior should be observed near the critical field, where the characteristic time $\tau$ diverges; in a dc-driven system, we should then expect $[3,9]$

$$
\langle x(t)\rangle \propto \frac{e^{-t / \tau}}{t^{\alpha}} \sim \begin{cases}t^{-\alpha} & \text { if } t \ll \tau, \\ e^{-t / \tau} & \text { if } t>\tau,\end{cases}
$$

for any observable coupled to the order parameter that tends to 0 under the stationary condition.

In the ac-driven vortex system, we propose a similar critical behavior with time measured by the number of applied cycles $N$. We chose as our observable the rate of change of $\chi^{\prime}$ per shaking cycle and we expect

$$
4 \pi\left(\chi^{\prime}(N)-\chi^{\prime}(N-1)\right)=A \frac{e^{-N / N_{\mathrm{ac}}\left(H_{\mathrm{sh}}\right)}}{N^{\alpha}},
$$

which implies

$$
\begin{aligned}
\frac{4 \pi}{A}\left(\chi_{\infty}^{\prime}-\chi^{\prime}(N)\right) & =\sum_{k=N+1}^{\infty} \frac{e^{-k / N_{\mathrm{ac}}}}{k^{\alpha}} \simeq \int_{N+1 / 2}^{\infty} d k \frac{e^{-k / N_{\mathrm{ac}}}}{k^{\alpha}} \\
& =N_{\mathrm{ac}}^{1-\alpha} \Gamma_{1-\alpha}\left(\frac{N+1 / 2}{N_{\mathrm{ac}}}\right),
\end{aligned}
$$

that in turn has been approximated, in the continuum limit, by the incomplete Gamma function $\Gamma_{v}(z)=\int_{z}^{\infty} t^{\nu-1} e^{-t} d t$. Here, shifting the lower limit down by $1 / 2$ compensates for truncation errors [41].

Figure 2(b) shows the evolution of $\Delta \chi^{\prime} / \Delta N$ with $N$ obtained from the data shown in Fig. 2(a), in double-logarithmic scale; the dashed line indicates the expected power-law behavior for the exponent $\alpha=1.07 \pm 0.01$, which best fits data for this particular frequency. Continuous lines in Fig. 2(a) are fits of the data with the function (3) using a unique exponent $\alpha$ and prefactor $A$ but different $N_{\mathrm{ac}}\left(H_{\mathrm{sh}}\right)$ [41], indicated by vertical arrows.

Equation (3) implies that, after normalization by $N_{\mathrm{ac}}^{1-\alpha}$, the data should scale when plotted as a function of $N / N_{\mathrm{ac}}$, for $N \gg 1$. Such scaling is shown in Fig. 3, where all data sets used in Fig. 2 are plotted. The data collapse is excellent up 


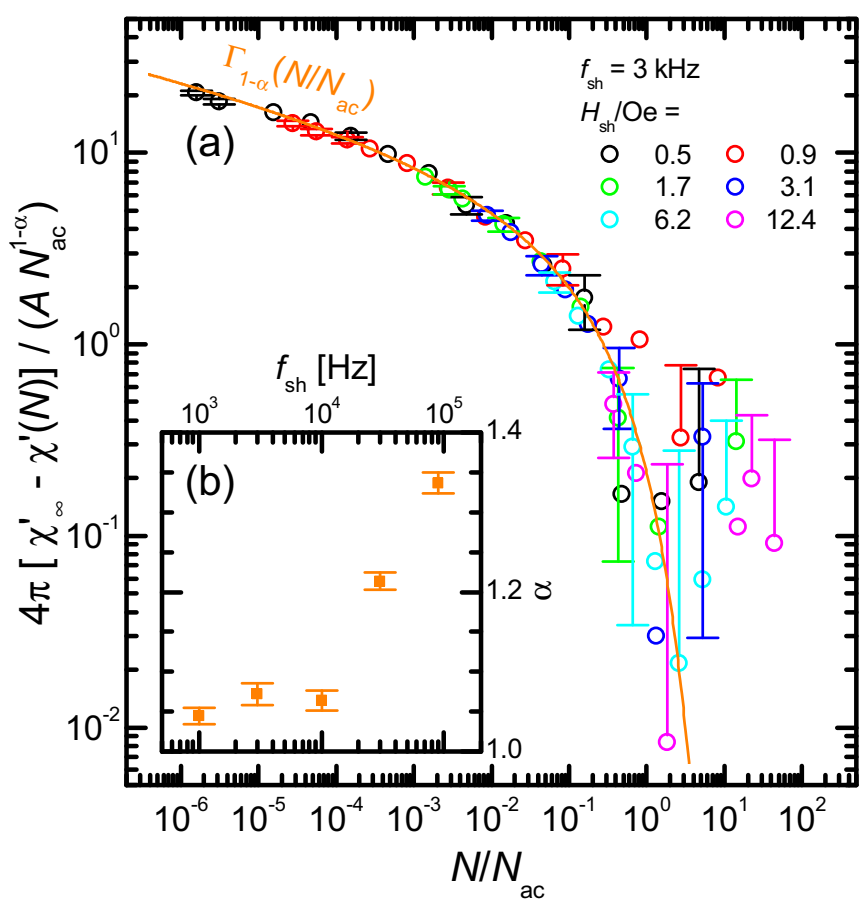

FIG. 3. (a) Scaling of the data shown in Fig. 2. The master curve is well represented by $\Gamma_{1-\alpha}\left(N / N_{\mathrm{ac}}\right)$, plotted with a continuous orange line. (b) The exponent $\alpha$ vs $f_{\text {sh. }}$.

to $N / N_{\mathrm{ac}} \simeq 1$, and the master curve $\Gamma_{1-\alpha}\left(N / N_{\mathrm{ac}}\right)$ (continuous line) represents the data very accurately. A similarly good scaling is obtained for shaking frequencies ranging from $f_{\mathrm{sh}}=$ 1 to $90 \mathrm{kHz}$ [41].

The critical exponent $\alpha$ is expected to be unique for each universality class of phase transitions. The resulting exponents $\alpha\left(f_{\mathrm{sh}}\right)$ are plotted in the inset of Fig. 3. A single frequency-independent $\alpha=1.02 \pm 0.02$ is obtained for $f_{\text {sh }}$ up to $10 \mathrm{kHz}$, but $\alpha$ increases at higher frequencies. Moreover, the characteristic number of cycles $N_{\mathrm{ac}}$ [indicated by vertical arrows in the example of Fig. 2(b)] is expected to diverge at a frequency-dependent critical field $H_{\mathrm{sh}}^{c}\left(f_{\mathrm{sh}}\right)$ as

$$
N_{\mathrm{ac}}\left(H_{\mathrm{sh}}, f_{\mathrm{sh}}\right)=N_{0}\left(H_{\mathrm{sh}}-H_{\mathrm{sh}}^{c}\left(f_{\mathrm{sh}}\right)\right)^{-\beta},
$$

with $\beta$ another critical exponent. By assigning weights to the expected models [Eqs. (3) and (4)], we were able to fit the whole set of data for all the shaking amplitudes and frequencies between 1 and $30 \mathrm{kHz}$, obtaining a single $\beta$ [41]. The main panel in Fig. 4 shows the transient number of cycles $N_{\mathrm{ac}}$ as a function of $H_{\mathrm{sh}}-H_{\mathrm{sh}}^{c}\left(f_{\mathrm{sh}}\right)$ for different $f_{\mathrm{sh}}$. All data collapse on a linear relationship with $\beta=2.4 \pm 0.4$, supporting the existence of an ac dynamic transition. The inset in Fig. 4 shows the resulting shaking critical field $H_{\mathrm{sh}}^{c}$ as a function of $f_{\mathrm{sh}}$.

A similar NEPT has been found in an ac-driven mean-field disordered spin model that mimics shaken granular systems [39]. In this simple model, the linear response $R$ converges to a stationary form whenever the system is assisted with strong enough ac fields. The transient time (measured in number of cycles) grows with decreasing amplitudes and diverges at a frequency-dependent critical amplitude $H_{\mathrm{sh}}^{c}$, similarly to what is reported in Fig. 4. This transition is, therefore, between a

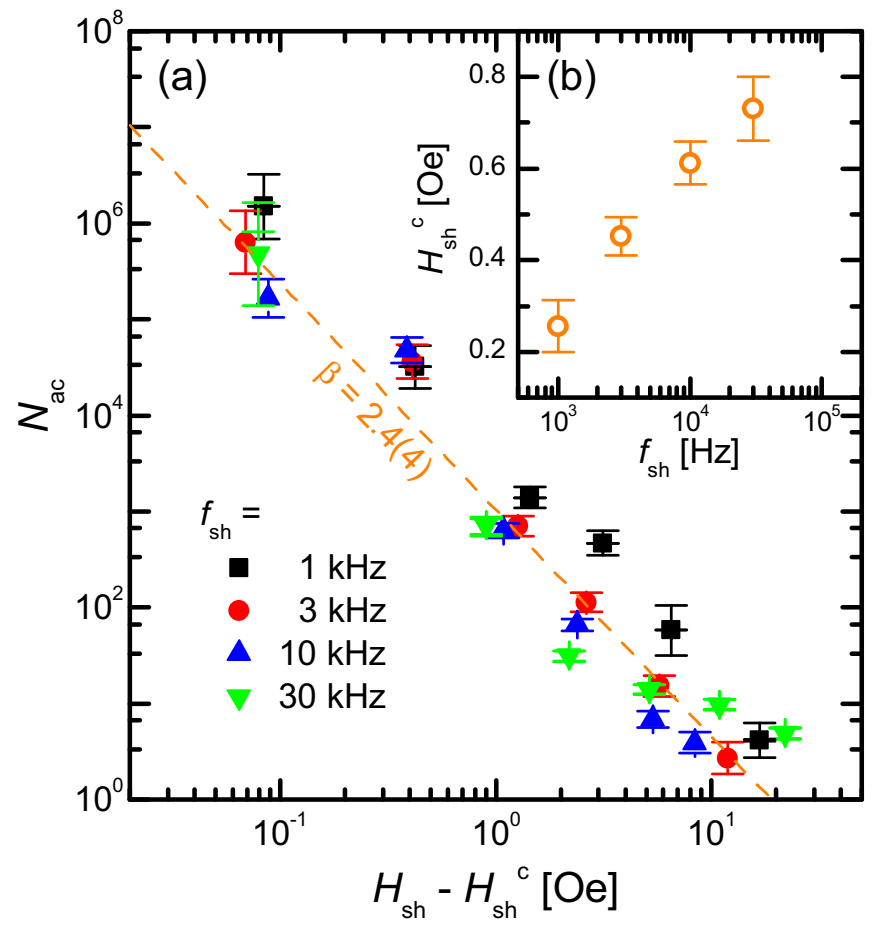

FIG. 4. (a) Dependence of $N_{\mathrm{ac}}$ with the distance from the critical field $H_{\mathrm{sh}}-H_{\mathrm{sh}}^{c}\left(f_{\mathrm{sh}}\right)$ in log-log scale; the slope for the resulting exponent $\beta$ [see Eq. (4)] is indicated with dashed line. (b) $H_{\mathrm{sh}}^{c}$ as a function of $f_{\mathrm{sh}}^{c}$.

viscous liquid and a glassy phase. The former is the steadystate phase for field amplitudes above $H_{\mathrm{sh}}^{c}\left(f_{\mathrm{sh}}\right)$.

Qualitatively, the same picture seems to hold in the present case: Reaching the memoryless steady state becomes more and more difficult when the strength of the shaking field decreases until it can no longer be done below the critical curve $H_{\mathrm{sh}}^{c}\left(f_{\mathrm{sh}}\right)$. A rough estimate of the vortex displacements during each shaking cycle indicates, under shaking fields of the order of the measured $H_{\mathrm{sh}}^{c}\left(f_{\mathrm{sh}}\right)$, that vortices move over distances that are smaller than the VL parameter and (on average) of the order of the coherence length (i.e., the typical pinning radius). Therefore, at lower shaking amplitudes, below the ac depinning, the system remains glassy. On the other hand, beyond the ac depinning, vortices move and reorganize into dynamic stationary states which lead to partially ordered vortex configurations. Whether this dynamic phase is characterized by steady fluctuating states [8], as suggested by molecular dynamic simulations in a simpler 2D model [20], is still an open question.

Although checking universal properties is notably difficult in dynamic phase transitions, we have succeeded in using critical slowing down and scaling arguments to characterize $\Delta \chi^{\prime} / \Delta N$ and the deviation of $\chi^{\prime}(N)$ from the asymptotic value $\chi_{\infty}^{\prime}\left(H_{\mathrm{sh}}, f_{\mathrm{sh}}\right)$, respectively. We found parameterindependent values of the critical exponents $\alpha$ and $\beta$, as expected, for low $f_{\mathrm{sh}}$ and all amplitudes. This accounts for the existence of a critical behavior, associated with a nonequilibrium phase transition driven by ac forces.

While universal independent values of the critical exponents are expected, deviations from constant $\alpha$ were mea- 
sured at high $f_{\text {sh }}$. This observation suggests a change in the universality class of the NEPT. The influence of viscous losses during shaking could promote a crossover from plastic to elastic dynamics at depinning. This possible explanation, supported by nonlinear ac susceptibility measurements [41], deserves further investigation.

In summary, we were able to experimentally observe a dynamic phase transition in a vortex system driven by ac forces, probably associated with ac depinning. The close similarity of vortex matter with other glassy systems opens the perspective to observe similar behavior in systems belonging to the broad class of elastic disordered media.

The authors acknowledge especially useful insights from V. Bekeris and thank M. Eskildsen, X. S. Ling, M. Mungan, S. Nagel, and S. Sastry for interesting discussions. This work was partially supported by Consejo Nacional de Investigaciones Científicas y Técnicas - Argentina (CONICET) and the University of Buenos Aires. L.F.C. thanks the KITP at UCSB for hospitality.
[1] H. Hinrichsen, Adv. Phys. 49, 815 (2000).

[2] L. F. Cugliandolo, C. R. Phys. 14, 685 (2013).

[3] C. Reichhardt and C. J. Olson Reichhardt, Rep. Prog. Phys. 80, 026501 (2017).

[4] P. J. Metaxas, J. P. Jamet, A. Mougin, M. Cormier, J. Ferré, V. Baltz, B. Rodmacq, B. Dieny, and R. L. Stamps, Phys. Rev. Lett. 99, 217208 (2007).

[5] V. Jeudy, A. Mougin, S. Bustingorry, W. Savero Torres, J. Gorchon, A. B. Kolton, A. Lemaître, and J.-P. Jamet, Phys. Rev. Lett. 117, 057201 (2016).

[6] T. Tybell, P. Paruch, T. Giamarchi, and J.-M. Triscone, Phys. Rev. Lett. 89, 097601 (2002).

[7] B. Ziegler, K. Martens, T. Giamarchi, and P. Paruch, Phys. Rev. Lett. 111, 247604 (2013).

[8] L. Corté, P. M. Chaikin, J. P. Gollub, and D. J. Pine, Nat. Phys. 4, 420 (2008).

[9] C. Reichhardt and C. J. Olson Reichhardt, Phys. Rev. Lett. 103, 168301 (2009).

[10] M.-C. Cha and H. A. Fertig, Phys. Rev. B 50, 14368 (1994).

[11] N. Nagaosa and Y. Tokura, Nat. Nanotechnol 8, 899 (2013).

[12] G. Blatter, M. V. Feigel'man, V. B. Geshkenbein, A. I. Larkin, and V. M. Vinokur, Rev. Mod. Phys. 66, 1125 (1994).

[13] A. B. Kolton, A. Rosso, T. Giamarchi, and W. Krauth, Phys. Rev. Lett. 97, 057001 (2006).

[14] T. Giamarchi, Disordered elastic media, in Encyclopedia of Complexity and Systems Science, edited by R. Meyers (Springer, New York, 2009).

[15] B. C. Chakrabarti and M. Acharyya, Rev. Mod. Phys. 71, 847 (1999).

[16] S. O. Valenzuela and V. Bekeris, Phys. Rev. Lett. 84, 4200 (2000).

[17] S. O. Valenzuela and V. Bekeris, Phys. Rev. Lett. 86, 504 (2001).

[18] G. Pasquini, D. Pérez Daroca, C. Chiliotte, G. S. Lozano, and V. Bekeris, Phys. Rev. Lett. 100, 247003 (2008).

[19] D. Pérez Daroca, G. S. Lozano, G. Pasquini, and V. Bekeris, Phys. Rev. B 81, 184520 (2010).

[20] D. Pérez Daroca, G. Pasquini, G. S. Lozano, and V. Bekeris, Phys. Rev. B 84, 012508 (2011).

[21] M. Marziali Bermúdez, M. R. Eskildsen, M. Bartkowiak, G. Nagy, V. Bekeris, and G. Pasquini, Phys. Rev. Lett. 115, 067001 (2015).

[22] P. Domenichini, C. P. Quinteros, M. Granada, S. Collin, J.-M. George, J. Curiale, S. Bustingorry, M. G.
Capeluto, and G. Pasquini, Phys. Rev. B 99, 214401 (2019).

[23] Y. Kawamura, S. Moriya, K. Ienaga, S. Kaneko, and S. Okuma, New J. Phys. 19, 093001 (2017).

[24] T. Giamarchi and P. Le Doussal, Phys. Rev. B 52, 1242 (1995).

[25] U. Yaron, P. L. Gammel, D. A. Huse, R. N. Kleiman, C. S. Oglesby, E. Bucher, B. Batlogg, D. J. Bishop, K. Mortensen, and K. N. Clausen, Nature (London) 376, 753 (1995).

[26] W. Henderson, E. Y. Andrei, M. J. Higgins, and S. Bhattacharya, Phys. Rev. Lett. 77, 2077 (1996).

[27] Y. Paltiel, E. Zeldov, Y. N. Myasoedov, H. Shtrikman, S. Bhattacharya, M. J. Higgins, Z. L. Xiao, E. Y. Andrei, P. L. Gammel, and D. J. Bishop, Nature (London) 403, 398 (2000).

[28] W. DeSorbo, Rev. Mod. Phys. 36, 90 (1964).

[29] A. Larkin and Y. Ovchinnikov, J. Low Temp. Phys. 34, 409 (1979).

[30] A. M. Troyanovski, M. van Hecke, N. Saha, J. Aarts, and P. H. Kes, Phys. Rev. Lett. 89, 147006 (2002).

[31] G. I. Menon, G. Ravikumar, M. J. Higgins, and S. Bhattacharya, Phys. Rev. B 85, 064515 (2012).

[32] Z. L. Xiao, E. Y. Andrei, P. Shuk, and M. Greenblatt, Phys. Rev. Lett. 85, 3265 (2000).

[33] S. C. Ganguli, H. Singh, G. Saraswat, R. Ganguly, V. Bagwe, P. Shirage, and A. T. P. Raychaudhuri, Sci. Rep. 5, 10613 (2015).

[34] C. J. van der Beek, V. B. Geshkenbein, and V. M. Vinokur, Phys. Rev. B 48, 3393 (1993).

[35] A. Campbell, J. Phys. C 4, 3186 (1971).

[36] B. Raes, C. C. de Souza Silva, A. V. Silhanek, L. R. E. Cabral, V. V. Moshchalkov, and J. Van de Vondel, Phys. Rev. B 90, 134508 (2014).

[37] C. S. Oglesby, E. Bucher, C. Kloc, and H. Hohl, J. Cryst. Growth 137, 289 (1994).

[38] H. M. Jaeger, S. R. Nagel, and R. P. Behringer, Rev. Mod. Phys. 68, 1259 (1996).

[39] L. Berthier, L. F. Cugliandolo, and J. L. Iguain, Phys. Rev. E 63, 051302 (2001).

[40] E. R. Louden, C. Rastovski, S. J. Kuhn, A. W. D. Leishman, L. DeBeer-Schmitt, C. D. Dewhurst, N. D. Zhigadlo, and M. R. Eskildsen, Phys. Rev. B 99, 060502(R) (2019).

[41] See Supplemental Material at http://link.aps.org/supplemental/ 10.1103/PhysRevB.101.060502 for details of the fitting procedure. 